\title{
TIGHTNESS OF THE RECENTERED MAXIMUM OF THE TWO-DIMENSIONAL DISCRETE GAUSSIAN FREE FIELD
}

\author{
MAURY BRAMSON* OFER ZEITOUNI ${ }^{\S}$
}

\begin{abstract}
We consider the maximum of the discrete two dimensional Gaussian free field (GFF) in a box, and prove that its maximum, centered at its mean, is tight, settling a long-standing conjecture. The proof combines a recent observation of [BDZ10] with elements from Br78] and comparison theorems for Gaussian fields. An essential part of the argument is the precise evaluation, up to an error of order 1 , of the expected value of the maximum of the GFF in a box. Related Gaussian fields, such as the GFF on a two-dimensional torus, are also discussed.
\end{abstract}

\section{INTRODUCTION}

We consider the discrete Gaussian Free Field (GFF) in a two-dimensional box of side $N$, with Dirichlet boundary conditions. That is, let $V_{N}=([0, N-$ $1] \cap \mathbb{Z})^{2}$ and $V_{N}^{o}=((0, N-1) \cap \mathbb{Z})^{2}$, and let $\left\{w_{m}\right\}_{m \geq 0}$ denote a simple random walk started in $V_{N}^{o}$ and killed at $\tau=\min \left\{m: w_{m} \in \partial V_{N}\right\}$ (that is, killed upon hitting the boundary $\left.\partial V_{N}=V_{N} \backslash V_{N}^{o}\right)$. For $x, y \in V_{N}$, define $G_{N}(x, y)=E^{x}\left(\sum_{m=0}^{\tau} \mathbf{1}_{w_{m}=y}\right)$, where $E^{x}$ denotes expectation with respect to the random walk started at $x$. The GFF is the zero-mean Gaussian field $\left\{\mathcal{X}_{z}^{N}\right\}_{z}$ indexed by $z \in V_{N}$ with covariance $G_{N}$.

Let $\mathcal{X}_{N}^{*}=\max _{z \in V_{N}} \mathcal{X}_{z}^{N}$. It was proved in [BDG01] that $\mathcal{X}_{N}^{*} /(\log N) \rightarrow c$ with $c=2 \sqrt{2 / \pi}$; the proof is closely related to the proof of the law of large numbers for the maximal displacement of a branching random walk in $\mathbb{R}$.

Let $M_{N}:=\mathcal{X}_{N}^{*}-E \mathcal{X}_{N}^{*}$. The goal of this paper is to prove the following.

Theorem 1.1. The sequence of random variables $\left\{M_{N}\right\}_{N \geq 1}$ is tight.

The statement in Theorem 1.1 has been a "folklore" conjecture for some time, and appears in print, e.g., as open problem \#4 in Ch08] (for an earlier appearance in print of a related conjecture, see [CLD01]). To the best of our knowledge, prior to the current paper, the sharpest result in this direction is due to [Ch08], who shows that the variance of $M_{N}$ is $o(\log N)$, and to [BDZ10], who show, building on an argument of [DH91], that Theorem 1.1 holds if one replaces $N$ by an appropriate deterministic sequence $\left\{N_{k}\right\}_{k \geq 1}$.

Date: September 16, 2010.

${ }^{*}$ Research partially supported by NSF grant number CCF-0729537.

${ }^{\S}$ Research partially supported by NSF grant number DMS-0804133 and by the Herman P. Taubman chair of Mathematics at the Weizmann Institute. 
In the same paper [BDZ10], it is shown that Theorem 1.1 holds as soon as one proves that, for an appropriate constant $C, E \mathcal{X}_{2 N}^{*} \leq E \mathcal{X}_{N}^{*}+C$ for all $N=2^{n}$ with $n$ integer. Theorem 1.1 thus follows immediately from the following theorem, which is our main result.

Theorem 1.2. With notation as above,

$$
E \mathcal{X}_{2^{n}}^{*}=c_{1} n-c_{2} \log n+O(1)
$$

with $c_{1}=2 \sqrt{2 / \pi} \log 2$ and $c_{2}=(3 / 4) \sqrt{2 / \pi}$.

One should note the striking similarity with the behavior of branching random walks (BRW), see Br78 (where branching Brownian motions are considered) and [ABR09]. The relation with (an imbedded) BRW is already apparent in BDG01, but the argument there is not sharp enough to allow for a control of the $\log n$ term in (1.1).

Our approach to the proof of Theorem 1.2 involves two main components. The first is a comparison argument (based on the Sudakov-Fernique inequality, see Lemma 2.1 below), that will allow us to quickly prove an upper bound in (1.1), and to relate $E \mathcal{X}_{2^{n}}^{*}$ to the expectation of the maximum of other Gaussian fields (and, in particular, to a version of the Gaussian Free Field on the torus, denoted $\left\{\mathcal{Y}_{x}^{N}\right\}_{x \in V_{N}}$ below, as well as to a modified version of branching random walk, denoted $\left\{\mathcal{S}_{x}^{N}\right\}_{x \in V_{N}}$ below). The second step consists of the analysis of the modified branching random walk $\mathcal{S}_{x}^{N}$, by properly modifying the second moment argument in [Br78] (see also [ABR09]).

Our results also provide an analog of Theorem 1.2 for the torus GFF $\left\{\mathcal{Y}_{x}^{N}\right\}_{x \in V_{N}}$, in Propositions 3.2 and 5.1, which is of interest in its own right. Intuitively, the model is the natural counterpart of the modified branching random walk $\left\{\mathcal{S}_{x}^{N}\right\}_{x \in V_{N}}$, which plays a central role in the proof of Theorem 1.2. We have not proved the analog of Theorem 1.1 for the torus GFF, which requires a modification of the argument in BDZ10.

The paper is structured as follows. In the next section, we recall a fundamental comparison between maxima of Gaussian fields; we then introduce the torus GFF, branching random walk, and modified branching random walk, and estimate their covariances. Section 3 is devoted to the proof of the upper bound in Theorem 1.2. The rest of the paper deals with the lower bound in Theorem 1.2. Section 4 reduces the proof of the lower bound to a lower bound on the maximum of a truncated version of the modified branching random walk introduced in Section 2. Section 5 reduces the proof of the latter to a lower bound on the maximum of the modified branching random walk over a subset of $V_{N}$. The proof of this bound is given in Section 6, using the second moment method. The proofs of some technical estimates, closely related to estimates in Br78, are sketched in the appendix.

Notation: throughout, the letter $C$ indicates a positive constant, independent of $N$, whose value may change from line to line. Positive constants that 
are fixed once and for all are denoted by the lower case $c$ with a subscript, for example $c_{5}$ or $c_{X}$.

\section{Preliminaries AND APPRoximations}

In this section, we recall a comparison tool between the maxima of different Gaussian fields and introduce Gaussian fields that approximate the GFF.

2.1. The Sudakov-Fernique inequality. The following inequality allows for the comparison of the expectation of the maxima of different Gaussian fields. For a proof, see [Fe75].

Lemma 2.1 (Sudakov-Fernique). Let $\mathcal{A}$ denote an arbitrary (finite) set, let $\left\{G_{\alpha}^{i}\right\}_{\alpha \in \mathcal{A}}, i=1,2$, denote two zero mean Gaussian fields and set $G_{i}^{*}=$ $\max _{\alpha \in \mathcal{A}} G_{\alpha}^{i}$. If

$$
E\left(G_{\alpha}^{1}-G_{\beta}^{1}\right)^{2} \geq E\left(G_{\alpha}^{2}-G_{\beta}^{2}\right)^{2}, \quad \text { for all } \alpha, \beta \in \mathcal{A},
$$

then

$$
E G_{1}^{*} \geq E G_{2}^{*} .
$$

In particular, if $\left\{G_{\alpha}\right\}_{\alpha \in \mathcal{A}}$ and $\left\{g_{\alpha}\right\}_{\alpha \in \mathcal{A}}$ are independent centered Gaussian fields, then one sees that

$$
E\left(\max _{\alpha \in \mathcal{A}}\left(G_{\alpha}+g_{\alpha}\right)\right) \geq E\left(\max _{\alpha \in \mathcal{A}} G_{\alpha}\right)
$$

a fact that is also easy to check without the Gaussian assumption.

2.2. The Torus GFF, Branching Random Walks, and Modified Branching Random Walks. We introduce several Gaussian fields with index set $V_{N}$ that will play a role in the proof of Theorem [1.2.

2.2.1. The Torus GFF. One of the drawbacks of working with the GFF is that its variance is not the same at all points of $V_{N}$. The Torus GFF (TGFF) $\left\{\mathcal{Y}_{z}^{N}\right\}_{z \in V_{N}}$ is a Gaussian field whose correlation structure resembles the GFF, but has the additional property that its variance is constant across $V_{N}$. To define it formally, for $x, y \in \mathbb{Z}^{2}$, write $x \sim_{N} y$ if $x-y \in(N \mathbb{Z})^{2}$. Similarly, for $B, B^{\prime} \subset V_{N}$, write $B \sim_{N} B^{\prime}$ if there exist integers $i, j$ so that $B^{\prime}=B+(i N, j N)$. Let $\tau^{\prime}$ denote an exponential random variable of parameter $1 / N^{2}$ and, with $\left\{w_{m}\right\}_{m \geq 0}$ denoting a simple random walk independent of $\tau^{\prime}$, define, for $x, y \in V_{N}$,

$$
\bar{G}_{N}(x, y)=E^{x}\left(\sum_{m=0}^{\tau^{\prime}} \mathbf{1}_{w_{m} \sim_{N} y}\right),
$$

where $E^{x}$ denotes expectation over both $\tau^{\prime}$ and the random walk started at $x$. That is, $\bar{G}_{N}$ is the Green function of a simple random walk on the torus of side $N$, killed at the independent exponential time $\tau^{\prime}$. The TGFF is the centered Gaussian process $\left\{\mathcal{Y}_{z}^{N}\right\}_{z \in V_{N}}$ with covariance $\bar{G}_{N}$. By construction, 
for $x, y \in V_{N}, E\left(\left(\mathcal{Y}_{x}^{N}\right)^{2}\right)=E\left(\left(\mathcal{Y}_{y}^{N}\right)^{2}\right)$, and an easy computation, using known properties of the Green function of two dimensional simple random walk, see, e.g., La91, reveals that

$$
\left|E\left(\left(\mathcal{Y}_{x}^{N}\right)^{2}\right)-\frac{2}{\pi} \log N\right| \leq C
$$

(Recall that, by our convention on constants, $C$ in (2.3) does not depend on $N$.) We define $\mathcal{Y}_{N}^{*}=\max _{z \in V_{N}} \mathcal{Y}_{z}^{N}$.

2.2.2. Branching Random Walks. In what follows, we consider $N=2^{n}$ for some positive integer $n$. For $k=0,1, \ldots, n$, let $\mathcal{B}_{k}$ denote the collection of subsets of $\mathbb{Z}^{2}$ consisting of squares of side $2^{k}$ with corners in $\mathbb{Z}^{2}$, let $\mathcal{B D}_{k}$ denote the subset of $\mathcal{B}_{k}$ consisting of squares of the form $\left(\left[0,2^{k}-1\right] \cap \mathbb{Z}\right)^{2}+$ $\left(i 2^{k}, j 2^{k}\right)$. Note that the collection $\mathcal{B D}_{k}$ partitions $\mathbb{Z}^{2}$ into disjoint squares. For $x \in V_{N}$, let $\mathcal{B}_{k}(x)$ denote those elements $B \in \mathcal{B}_{k}$ with $x \in B$. Define similarly $\mathcal{B D}_{k}(x)$. Note that the set $\mathcal{B D}_{k}(x)$ contains exactly one element, whereas $\mathcal{B}_{k}(x)$ contains $2^{2 k}$ elements.

Let $\left\{a_{k, B}\right\}_{k \geq 0, B \in \mathcal{B D}_{k}}$ denote an i.i.d. family of standard Gaussian random variables. The BRW $\left\{\mathcal{R}_{z}^{N}\right\}_{z \in V_{N}}$ is defined by

$$
\mathcal{R}_{z}^{N}=\sum_{k=0}^{n} \sum_{B \in \mathcal{B D}_{k}(z)} a_{k, B}
$$

We again define $\mathcal{R}_{N}^{*}=\max _{z \in V_{N}} \mathcal{R}_{z}^{N}$.

2.2.3. Modified Branching Random Walks. We continue to consider $N=2^{n}$ for some positive integer $n$ and again employ the notation $\mathcal{B}_{k}$ and $\mathcal{B}_{k}(x)$. Let $\mathcal{B}_{k}^{N}$ denote the collection of subsets of $\mathbb{Z}^{2}$ consisting of squares of side $2^{k}$ with lower left corner in $V_{N}$. Let $\left\{b_{k, B}\right\}_{k \geq 0, B \in \mathcal{B}_{k}^{N}}$ denote an i.i.d. family of centered Gaussian random variables of variance $2^{-2 k}$, and define

$$
b_{k, B}^{N}= \begin{cases}b_{k, B}, & B \in \mathcal{B}_{k}^{N} \\ b_{k, B^{\prime}}, & B \sim_{N} B^{\prime} \in \mathcal{B}_{k}^{N} .\end{cases}
$$

The modified branching random walk (MBRW) $\left\{\mathcal{S}_{z}^{N}\right\}_{z \in V_{N}}$ is defined by

$$
\mathcal{S}_{z}^{N}=\sum_{k=0}^{n} \sum_{B \in \mathcal{B}_{k}(z)} b_{k, B}^{N} .
$$

Note that, by construction, $E\left(\left(\mathcal{R}_{z}^{N}\right)^{2}\right)=E\left(\left(\mathcal{S}_{z}^{N}\right)^{2}\right)=n+1$. We again define $\mathcal{S}_{N}^{*}=\max _{z \in V_{N}} \mathcal{S}_{z}^{N}$.

2.2.4. Geometric distances. The following are several notions of distances between points in $V_{N}$. First, $\|\cdot\|$ denotes the Euclidean norm, while $\|\cdot\|_{\infty}$ denotes the $\ell^{\infty}$ norm. Thus, for $x, y \in V_{N},\|x-y\|$ and $\| x-\left.y\right|_{\infty}$ induce metrics with

$$
\|x-y\|_{\infty} \leq\|x-y\| \leq \sqrt{2}\|x-y\|_{\infty} .
$$


We also need to consider distances on the torus determined by $V_{N}$. Those are defined by

$$
d^{N}(x, y)=\min _{z: z \sim_{N} y}\|x-z\|, \quad d_{\infty}^{N}(x, y)=\min _{z: z \sim_{N} y}\|x-z\|_{\infty} .
$$

2.3. Covariance comparisons. We collect in this subsection some basic facts concerning the covariances of the Gaussian fields introduced earlier. For a centered Gaussian field $\left\{G_{z}\right\}$, we write $\mathbf{R}_{G}(x, y)=E\left(G_{x} G_{y}\right)$ for its covariance function. Thus, for example, the covariance function of the GFF (on $V_{N}$ ) is denoted by $\mathbf{R}_{\mathcal{X}^{N}}$.

The following is an estimate on $\mathbf{R}_{\mathcal{Y}^{N}}, \mathbf{R}_{\mathcal{S}^{N}}$ and $\mathbf{R}_{\mathcal{X}^{N}}$.

Lemma 2.2. There exists a constant $C$ so that, with $N=2^{n}$, the following estimates hold: for any $x, y \in V_{N}$,

$$
\left|\mathbf{R}_{\mathcal{Y}^{N}}(x, y)-\frac{2 \log 2}{\pi}\left(n-\log _{2} d^{N}(x, y)\right)\right| \leq C
$$

and

$$
\left|\mathbf{R}_{\mathcal{S}^{N}}(x, y)-\left(n-\log _{2} d^{N}(x, y)\right)\right| \leq C .
$$

Further, for any $x, y \in V_{N}+(2 N, 2 N)$,

$$
\left|\mathbf{R}_{\mathcal{X}^{4 N}}(x, y)-\frac{2 \log 2}{\pi}\left(n-\left(\log _{2}\|x-y\|\right)_{+}\right)\right| \leq C .
$$

Proof. We begin with the estimate (2.5) concerning the MBRW. For $x=$ $\left(x_{1}, x_{2}\right)$ and $y=\left(y_{1}, y_{2}\right)$, write, for $i=1,2, t_{i}(x, y)=\min \left(\left|x_{i}-y_{i}\right|, \mid x_{i}-\right.$ $\left.y_{i}-N|,| x_{i}-y_{i}+N \mid\right)$. One then has

$$
\begin{aligned}
\mathbf{R}_{\mathcal{S}^{N}}(x, y) & =\sum_{k=\left\lceil\log _{2}\left(d_{\infty}^{N}(x, y)+1\right)\right\rceil}^{n} 2^{-2 k}\left[2^{k}-t_{1}(x, y)\right] \cdot\left[2^{k}-t_{2}(x, y)\right] \\
(2.7) & =\sum_{k=\left\lceil\log _{2}\left(d_{\infty}^{N}(x, y)+1\right)\right\rceil}^{n}\left(1-\frac{t_{1}(x, y)}{2^{k}}-\frac{t_{2}(x, y)}{2^{k}}+\frac{t_{1}(x, y) t_{2}(x, y)}{4^{k}}\right) .
\end{aligned}
$$

Because $a+b-a b \geq 0$ for $0 \leq a, b \leq 1$, we get that

$$
\mathbf{R}_{\mathcal{S}^{N}}(x, y) \leq n-\log _{2}\left(d_{\infty}^{N}(x, y)+1\right)+2 \leq n-\log _{2}\left(d^{N}(x, y)+1\right)+3 .
$$

On the other hand, using that $a+b-a b \leq a+b$ for $a, b \geq 0$, we get that

$$
\begin{array}{ll}
\mathbf{R}_{\mathcal{S}^{N}}(x, y) & \geq n-\log _{2}\left(d_{\infty}^{N}(x, y)+1\right)-\sum_{k=\left\lceil\log _{2}\left(d_{\infty}^{N}(x, y)+1\right)\right\rceil}^{n} 2^{-(k-1)} d_{\infty}^{N}(x, y) \\
(2.9) & \geq n-\log _{2}\left(d^{N}(x, y)+1\right)-C .
\end{array}
$$

Combining (2.8) and (2.9) yields the claimed estimate on $\mathbf{R}_{\mathcal{S}^{N}}$.

We next prove the estimate (2.6). Note that for $x, y \in V_{N}+(2 N, 2 N)$,

$$
\mathbf{R}_{\mathcal{X}^{4 N}}(x, y)=P^{x}\left(\tau_{y} \leq \tau_{4 N}\right) \mathbf{R}_{\mathcal{X}^{4 N}}(y, y),
$$


where $\tau_{y}=\min \left\{m \geq 0: w_{m}=y\right\}$ and $\tau_{4 N}=\min \left\{m \geq 0: w_{m} \notin V_{4 N}\right\}$. Using, e.g., La91, Exercise 1.6.8],

$$
P^{x}\left(\tau_{y} \leq \tau_{4 N}\right)=\left(1-\frac{(\log \|x-y\|)_{+}}{n \log 2}\right)+\frac{O(1)}{n} .
$$

Moreover, for $x \in V_{N}+(2 N, 2 N)$,

$$
\mathbf{R}_{\mathcal{X}^{4 N}}(x, x)=\frac{2 \log 2}{\pi} n+O(1)
$$

see, e.g., Ch08. Combining these estimates yields (2.6).

The estimate on $\mathbf{R}_{\mathcal{Y}^{N}}$ in (2.4) requires more work but is still straight forward. Recall the simple random walk $\left\{w_{m}\right\}$ and, for $y \in V_{N}$, denote by $[y]_{N}=\left\{z \in V_{N}: z \sim_{N} y\right\}$ the collection of points in $\mathbb{Z}^{2}$ identified with $y$ for the torus. Then, by the Markov property and the memoryless property of the exponential distribution,

$$
\begin{aligned}
\mathbf{R}_{\mathcal{Y}^{N}}(x, y) & =E\left(\mathcal{Y}_{y}^{N}\right)^{2} P^{x}\left(\left\{w_{m}\right\} \text { hits }[y]_{N} \text { before } \tau^{\prime}\right) \\
& =\frac{2 \log 2}{\pi} n P^{x}\left(\left\{w_{m}\right\} \text { hits }[y]_{N} \text { before } \tau^{\prime}\right)+O(1),
\end{aligned}
$$

where we recall that $\tau^{\prime}$ denotes a geometric random variable of mean $N^{2}$ and we used (2.3) in the second equality.

Let $\eta$ denote the hitting time of the boundary of a (Euclidean) ball of radius $N / 2$ around $x$, that is

$$
\eta=\min \left\{m \geq 0:\left\|w_{m}-x\right\| \geq N / 2\right\} .
$$

Let $\tau_{y}$ denote the hitting time of $[y]_{N}$, that is

$$
\tau_{y}=\min \left\{m \geq 0: w_{m} \in[y]_{N}\right\} .
$$

Note that the probability in the right side of (2.11) is $P^{x}\left(\tau_{y}<\tau^{\prime}\right)$. We have

$$
\begin{array}{ll}
P^{x}\left(\tau_{y}<\tau^{\prime}\right) & =P^{x}\left(\tau_{y}<\eta\right)+P^{x}\left(\tau_{y}<\tau^{\prime}, \tau_{y} \geq \eta\right)-P^{x}\left(\tau_{y}<\eta, \tau_{y} \geq \tau^{\prime}\right) \\
(2.12) & =: P_{1}+P_{2}-P_{3} .
\end{array}
$$

By standard estimates for two dimensional simple random walk, see again e.g., [La91, Exercise 1.6.8],

$$
\left|P_{1}-\left[n-\log _{2}|x-y|\right]_{+} / n\right| \leq C / n
$$

and, using the memoryless property of the exponential distribution,

$$
P_{2} \leq \max _{z:\left\|z-[y]_{N}\right\| \geq N / 4} P^{z}\left(\tau_{y} \leq \tau^{\prime}\right) \leq C / n .
$$

To estimate $P_{3}$, we use the fact (see e.g., Ch08, Lemma 10.4]) that, for all $m \geq 1$,

$$
P^{x}\left(w_{m} \in[y]_{N}\right) \leq \begin{cases}\frac{C}{m} e^{-\left(d^{N}(x, y)\right)^{2} / 4 m}, & m \leq N^{2} \\ \frac{C}{N^{2}}, & m>N^{2}\end{cases}
$$


Write $P_{m}(x, z):=P^{x}\left(w_{m} \in[z]_{N}\right)$. Then, again using the memoryless property of the exponential distribution and the Markov property of the simple random walk,

$$
P_{3}=P^{x}\left(\tau^{\prime} \leq \tau_{y}<\eta\right) \leq \frac{C}{N^{2}} \sum_{m=1}^{\infty} e^{-m / N^{2}} \sum_{z \in V_{N}} P_{m}(x, z) P^{z}\left(\tau_{y}<\eta\right) .
$$

We split the sum in the right side of (2.15) into three parts, according to the range of $m$ in the summation, writing $P_{3}=P_{3,1}+P_{3,2}+P_{3,3}$, with the terms in the right side determined according to $m \leq N^{2} / n, m \in\left(N^{2} / n, N^{2}\right)$ or $m \geq N^{2}$. We have

$$
P_{3,1}=\frac{C}{N^{2}} \sum_{m=1}^{N^{2} / n} e^{-m / N^{2}} \sum_{z \in V_{N}} P_{m}(x, z) P^{z}\left(\tau_{y}<\eta\right) \leq \frac{C}{N^{2}} \sum_{m=1}^{N^{2} / n} e^{-m / N^{2}} \leq \frac{C}{n} .
$$

Next, consider $P_{3,3}$ : we have, using (2.14) in the first inequality and standard estimates for simple random walk in the second, see [La91, Exercise 1.6.8],

$$
\begin{aligned}
P_{3,3} & =\frac{C}{N^{2}} \sum_{m=N^{2}}^{\infty} e^{-m / N^{2}} \sum_{z \in V_{N}} P_{m}(x, z) P^{z}\left(\tau_{y}<\eta\right) \\
& \leq \frac{C}{N^{4}} \sum_{m=N^{2}}^{\infty} e^{-m / N^{2}} \sum_{z \in V_{N}} P^{z}\left(\tau_{y}<\eta\right) \\
& \leq \frac{C}{N^{4}} \sum_{m=N^{2}}^{\infty} e^{-m / N^{2}} \sum_{z \in V_{N}}\left(\frac{n-\log _{2} d^{N}(z, y)}{n}\right) \\
& \leq \frac{C}{N^{2}} \sum_{z \in V_{N}}\left(\frac{n-\log _{2} d^{N}(z, y)}{n}\right) \\
& \leq \frac{C}{N^{2}} \sum_{r=1}^{n} 2^{2 r}\left(1-\frac{r}{n}\right) \\
& =C \sum_{r=1}^{n} 2^{2(r-n)}\left(1-\frac{r}{n}\right)=\frac{C}{n} \sum_{k=1}^{n} k 2^{-2 k} \leq \frac{C}{n} .
\end{aligned}
$$

It remains to estimate $P_{3,2}$. Note first that, for $x$ and $y$ fixed and each integer part of the value of $d^{N}(x, z)$, there are at most $C r$ many possible points $z \in V_{N}$ with $d^{N}(y, z) \in[r, 2 r]$. Also, due to (2.14), we can write

$$
\begin{aligned}
P_{3,2} & \leq \frac{C}{n}+\frac{C}{N^{2}} \sum_{m=N^{2} / n}^{N^{2}} e^{-m / N^{2}} \sum_{z \in V_{N}, d^{N}(x, z) \leq d_{m}} P_{m}(x, z) P^{z}\left(\tau_{y}<\eta\right) \\
& =: \frac{C}{n}+\frac{C}{N^{2}} \sum_{m=N^{2} / n}^{N^{2}} e^{-m / N^{2}} P_{3,2, m}
\end{aligned}
$$


where $d_{m}=\sqrt{m \log \log m} \wedge \sqrt{2} N$. By summing radially (so that the index $k$ runs over the possible integer parts of $d^{N}(x, z)$ and $n-\ell$ runs over the possible integer parts of $\log _{2} d^{N}(y, z)$ ), we can estimate $P_{3,2, m}$ (using (2.13) for the estimate $P_{m}(x, z) \leq C m^{-1} e^{-k^{2} / 4 m}$ and (2.14) for the estimate $\left.P^{z}\left(\tau_{y}<\eta\right) \leq C \ell / n\right)$ by

$$
\frac{C}{n m} \sum_{k=1}^{d_{m}} e^{-k^{2} / 4 m} \sum_{\ell=1}^{n} \ell 2^{n-\ell} \leq \frac{C N}{n m} \sum_{k=1}^{d_{m}} e^{-k^{2} / 4 m} \leq \frac{C N}{n \sqrt{m}} .
$$

Substituting in the expression for $P_{3,2}$, we get

$$
P_{3,2} \leq \frac{C}{n}+\frac{C N}{N^{2} n} \sum_{m=N^{2} / n}^{N^{2}} \frac{e^{-m / N^{2}}}{\sqrt{m}} \leq \frac{C}{n} .
$$

Combining the estimates on $P_{3,1}, P_{3,2}$ and $P_{3,3}$ and substituting in (2.15) shows that $P_{3} \leq C / n$. Together with (2.11), (2.12) and the estimates on $P_{1}$ and $P_{2}$, this completes the proof of the claimed estimate on $\mathbf{R}_{\mathcal{Y}^{N}}$ and hence of the lemma.

\section{THE UPPER BOUND}

Our goal in this section is to provide the upper bound in Theorem 1.2. this is achieved in Proposition 3.2 below. We begin by relating the maxima of the GFF and the TGFF with the MBRW.

Lemma 3.1. Let $\left\{g_{z}\right\}_{z \in V_{N}}$ denote a collection of i.i.d. standard Gaussian random variables. Then, there exists a constant $C_{1}$ so that

$$
\max \left(E \mathcal{X}_{N}^{*}, E \mathcal{Y}_{N}^{*}\right) \leq \sqrt{\frac{2 \log 2}{\pi}} E\left(\max _{z \in V_{N}}\left(\mathcal{S}_{z}^{N}+C_{1} g_{z}\right)\right)
$$

Proof. We give the argument for the GFF; the argument for the TGFF is similar. Note first that, by the definitions and an application of Lemma 2.1.

$$
E\left(\mathcal{X}_{N}^{*}\right) \leq E\left(\max _{z \in V_{N}+(2 N, 2 N)} \mathcal{X}_{z}^{4 N}\right) .
$$

On the other hand, writing $x_{N}=x+(2 N, 2 N), y_{N}=y+(2 N, 2 N)$ for $x, y \in V_{N}$ and using (2.6) of Lemma 2.2, we have

$$
E\left(\left(\mathcal{X}_{x_{N}}^{4 N}-\mathcal{X}_{y_{N}}^{4 N}\right)^{2}\right) \leq \frac{2 \log 2}{\pi} E\left(\left(\mathcal{S}_{x}^{N}-\mathcal{S}_{y}^{N}\right)^{2}\right)+C .
$$

Another application of Lemma 2.1, together with (3.2), completes the proof of (3.1) for the GFF.

It follows from [Br78] and [ABR09] that

$$
E \mathcal{R}_{N}^{*}=2 \sqrt{\log 2} n-\frac{3}{4 \sqrt{\log 2}} \log n+O(1) .
$$

(The statement in $\mathrm{Br} 78$ is given for branching Brownian motions, but the argument given there applies to our BRW as well.) This fact, together with 
Lemmas 2.1 and 3.1, yields the following upper bound on the GFF and the TGFF.

Proposition 3.2. There exists a constant $C_{2}$ such that

$$
\max \left(E \mathcal{X}_{N}^{*}, E \mathcal{Y}_{N}^{*}\right) \leq 2 \log 2 \sqrt{\frac{2}{\pi}} n-\frac{3}{4} \sqrt{\frac{2}{\pi}} \log n+C_{2} .
$$

Proof. By construction, for $x, y \in V_{N}, E\left(\left(\mathcal{R}_{x}^{N}\right)^{2}\right)=E\left(\left(\mathcal{S}_{x}^{N}\right)^{2}\right)$ and $\mathbf{R}_{\mathcal{R}^{N}}(x, y) \leq$ $\mathbf{R}_{\mathcal{S}^{N}}(x, y)+C$. By Lemma 2.1, this yields the existence of a positive integer $\bar{C}_{1}$ such that, with $C_{1}$ and $g_{z}$ as in Lemma 3.1,

$$
E\left(\max _{z \in V_{N}}\left(\mathcal{S}_{z}^{N}+C_{1} g_{z}\right)\right) \leq E\left(\max _{z \in V_{N}}\left(\mathcal{R}_{z}^{N}+\bar{C}_{1} g_{z}\right)\right) .
$$

Note however that, by construction,

$$
E\left(\max _{z \in V_{N}}\left(\mathcal{R}_{z}^{N}+\bar{C}_{1} g_{z}\right)\right) \leq E\left(\mathcal{R}_{2}^{*} \bar{C}_{1}^{2} N\right) .
$$

Combining this with (3.4), (3.6) and Lemma 3.1 completes the proof of (3.5) and hence of Proposition 3.2 .

\section{THE LOWER BOUND: PRELIMINARIES}

In this section, we bound from below the expected maxima of the GFF and the TGFF by an appropriate truncation of the MBRW. An analysis of the latter, provided in Sections 5 and 6, will then complete the proof of Theorem 1.2 .

We begin by introducing the truncation of the MBRW alluded to above. Recall that

$$
\mathcal{S}_{z}^{N}=\sum_{k=0}^{n} \sum_{B \in \mathcal{B}_{k}(z)} b_{k, B}^{N} .
$$

For a non-negative integer $k_{0} \leq n$, define

$$
\mathcal{S}_{z}^{N, k_{0}}=\sum_{k=k_{0}}^{n} \sum_{B \in \mathcal{B}_{k}(z)} b_{k, B}^{N},
$$

and write $\mathcal{S}_{N, k_{0}}^{*}=\max _{z \in V_{N}} \mathcal{S}_{z}^{N, k_{0}}$. Clearly, $\mathcal{S}^{N, 0}=\mathcal{S}^{N}$. Define, for $x, y \in$ $V_{N}, \rho_{N, k_{0}}(x, y)=E\left(\left(\mathcal{S}_{x}^{N, k_{0}}-\mathcal{S}_{y}^{N, k_{0}}\right)^{2}\right)$. The following are basic properties of $\rho_{N, k_{0}}$.

Lemma 4.1. The function $\rho_{N, k_{0}}$ has the following properties.

$$
\begin{aligned}
& \rho_{N, k_{0}}(x, y) \text { decreases in } k_{0} . \\
& \limsup _{k_{0} \rightarrow \infty} \limsup _{N \rightarrow \infty} \sup _{x, y \in V_{N}: d_{N}(x, y) \leq 2 \sqrt{k_{0}}} \rho_{N, k_{0}}(x, y)=0 .
\end{aligned}
$$

There is a function $g: \mathbb{Z}_{+} \rightarrow \mathbb{R}_{+}$so that $g\left(k_{0}\right) \rightarrow_{k_{0} \rightarrow \infty} \infty$ and, for $x, y \in V_{N}$ with $d^{N}(x, y) \geq 2^{\sqrt{k_{0}}}$, $\rho_{N, k_{0}}(x, y) \leq \rho_{N, 0}(x, y)-g\left(k_{0}\right), \quad n>k_{0}$. 
Proof. As in (2.7) and employing the same notation, we have, for $x \neq y$,

$$
\begin{aligned}
& \sum_{N, k_{0}}(x, y) \\
& \quad \sum_{k=\left\lceil\log _{2}\left(d_{\infty}^{N}(x, y)+1\right)\right\rceil \vee k_{0}}^{n} 2\left(\frac{t_{1}(x, y)}{2^{k}}+\frac{t_{2}(x, y)}{2^{k}}-\frac{t_{1}(x, y) t_{2}(x, y)}{4^{k}}\right) \\
& \quad+2\left(\left\lceil\log _{2}\left(d_{\infty}^{N}(x, y)+1\right)\right\rceil-k_{0}\right)_{+} .
\end{aligned}
$$

All properties follow at once from this representation. Indeed, (4.1) and (4.2) are immediate whereas, to see (4.3), note that, for $\log _{2} d_{\infty}^{N}(x, y) \geq \sqrt{k_{0}}-1$,

$$
\rho_{N, 0}(x, y)-\rho_{N, k_{0}}(x, y) \geq \sqrt{k_{0}}-1 .
$$

An immediate corollary of Lemmas 2.1, 2.2 and 4.1 is the following domination by the TGFF of a truncated MBRW.

Corollary 4.2. There exists a constant $k_{0}$ such that, for all $N=2^{n}$ large and all $x, y \in V_{N}$,

$$
\frac{2 \log 2}{\pi} \rho_{N, k_{0}}(x, y) \leq E\left(\left(\mathcal{Y}_{x}^{N}-\mathcal{Y}_{y}^{N}\right)^{2}\right) .
$$

In particular,

$$
E \mathcal{Y}_{N}^{*} \geq \sqrt{\frac{2 \log 2}{\pi}} E \mathcal{S}_{N, k_{0}}^{*}
$$

We also need a comparison between the maxima of the GFF and of the MBRW. Note that

$$
\mathcal{X}_{N}^{*} \geq \max _{z \in V_{N / 4}+(N / 2, N / 2)} \mathcal{X}_{z}^{N}
$$

On the other hand, for $x, y \in V_{N / 4}$, we have, with the same proof as that of (3.3),

$$
E\left(\left(\mathcal{X}_{x+(N / 2, N / 2)}^{N}-\mathcal{X}_{y+(N / 2, N / 2)}^{N}\right)^{2}\right) \geq \frac{2 \log 2}{\pi} E\left(\left(\mathcal{S}_{x}^{N / 4}-\mathcal{S}_{y}^{N / 4}\right)^{2}\right)-C
$$

Using again Lemmas 2.1 and 4.1, we get the following domination by the GFF of a truncated MBRW.

Corollary 4.3. There exists a constant $k_{0}$ such that, for all $N=2^{n}$ large, and all $x, y \in V_{N}$,

$$
E \mathcal{X}_{N}^{*} \geq \sqrt{\frac{2 \log 2}{\pi}} E \mathcal{S}_{N / 4, k_{0}}^{*} .
$$




\section{A LOWER BOUnd FOR THE TRUNCATEd MBRW}

In this section, we present the proof of the lower bound in Theorem 1.2 and an analogous bound for the TGFF. That is, we prove the following.

Proposition 5.1. The following holds:

$$
E \mathcal{X}_{2^{n}}^{*} \geq c_{1} n-c_{2} \log n+O(1), \quad E \mathcal{Y}_{2^{n}}^{*} \geq c_{1} n-c_{2} \log n+O(1),
$$

with $c_{1}=2 \sqrt{2 / \pi} \log 2$ and $c_{2}=(3 / 4) \sqrt{2 / \pi}$.

In view of Corollaries 4.2 and 4.3 , it is immediate that Proposition 5.1 follows from the following proposition, whose proof will take the rest of this section and the next one.

Proposition 5.2. There exists a function $f: \mathbb{Z}_{+} \rightarrow \mathbb{R}_{+}$such that, for all $N \geq 2^{2 k_{0}}$,

$$
E \mathcal{S}_{N, k_{0}}^{*} \geq(2 \sqrt{\log 2}) n-(3 /(4 \sqrt{\log 2})) \log n-f\left(k_{0}\right) .
$$

When proving Proposition 5.2, it will be more convenient to restrict the maximum in the definition of $S_{N, k_{0}}^{*}$ to a subset of $V_{N}$. Toward this end, set $V_{N}^{\prime}=V_{N / 2}+(N / 4, N / 4) \subset V_{N}$ and define

$$
\tilde{S}_{N, k_{0}}^{*}=\max _{z \in V_{N}^{\prime}} S_{z}^{N, k_{0}}, \quad \tilde{S}_{N}^{*}=\tilde{S}_{N, 0}^{*} .
$$

The main ingredient in the proof of Proposition 5.2 is a lower bound on the upper tail of the distribution of $\tilde{\mathcal{S}}_{N, 0}^{*}$, as given in Proposition 5.3 below.

Proposition 5.3. Let $A_{n}=(2 \sqrt{\log 2}) n-(3 /(4 \sqrt{\log 2})) \log n$. There exists a constant $\delta_{0} \in(0,1)$ such that, for all $N$,

$$
P\left(\tilde{\mathcal{S}}_{N}^{*} \geq A_{n}\right) \geq \delta_{0} .
$$

The proof of Proposition 5.3 is technically involved and is deferred to Section 6. In the rest of this section, we show how Proposition 5.2 follows from Proposition 5.3 .

Proof of Proposition 5.2 (assuming Proposition 5.3) Our plan is to show that the left tail of $\tilde{S}_{N}^{*}$ is decreasing exponentially fast; together with the bound (5.3), this will imply (5.2) with $k_{0}=0$. At the end of the proof, we show how the bound for $k_{0}>0$ follows from the case $k_{0}=0$. In order to show the exponential decay, we compare $\tilde{S}_{N}^{*}$, after appropriate truncation, to four independent copies of the maximum over smaller boxes, and then iterate.

For $i=1,2,3,4$, introduce the four sets $W_{N, i}=[0, N / 32)^{2}+z_{i}$ where $z_{1}=(N / 4, N / 4), z_{2}=(23 N / 32, N / 4), z_{3}=(N / 4,23 N / 32)$ and $z_{4}=$ $(23 N / 32,23 N / 32)$. (We have used here that $3 / 4-1 / 32=23 / 32$.) Note that $\cup_{i} W_{N, i} \subset V_{N}$, and that these sets are $N / 4$-separated, that is, for $i \neq j$,

$$
\min _{z \in W_{N, i}, z^{\prime} \in W_{N, j}} d_{\infty}^{N}(x, y)>N / 4
$$


Recall the definition of $\mathcal{S}_{z}^{N}$ and define, for $n>6$,

$$
\overline{\mathcal{S}}_{z}^{N}=\sum_{k=0}^{n-6} \sum_{B \in \mathcal{B}_{k}(z)} b_{k, B}^{N}
$$

note that

$$
\mathcal{S}_{z}^{N}-\overline{\mathcal{S}}_{z}^{N}=\sum_{j=0}^{5} \sum_{B \in \mathcal{B}_{n-j}(z)} b_{n-j, B}^{N}
$$

Our first task is to bound the probability that $\max _{z \in V_{N}}\left(\mathcal{S}_{z}^{N}-\overline{\mathcal{S}}_{z}^{N}\right)$ is large. This will be achieved by applying Fernique's criterion in conjunction with Borell's inequality. We introduce some notation. Let $m(\cdot)=m_{N}(\cdot)$ denote the uniform probability measure on $V_{N}$ (i.e., the counting measure normalized by $\left.\left|V_{N}\right|\right)$ and let $g:(0,1] \rightarrow \mathbb{R}_{+}$be the function defined by

$$
g(t)=(\log (1 / t))^{1 / 2} .
$$

Set $\mathbf{G}_{z}^{N}=\mathcal{S}_{z}^{N}-\overline{\mathcal{S}}_{z}^{N}$ and

$$
B(z, \epsilon)=\left\{z^{\prime} \in V_{N}: E\left(\left(\mathbf{G}_{z}^{N}-\mathbf{G}_{z^{\prime}}^{N}\right)^{2}\right) \leq \epsilon^{2}\right\} .
$$

Then, Fernique's criterion, see [Ad90, Theorem 4.1], implies that, for some universal constant $K \in(1, \infty)$,

$$
E\left(\max _{z \in V_{N}} \mathbf{G}_{z}^{N}\right) \leq K \sup _{z \in V_{N}} \int_{0}^{\infty} g(m(B(z, \epsilon))) d \epsilon .
$$

For $n \geq 6$, we have, in the notation of Lemma 4.1,

$$
E\left(\left(\mathbf{G}_{z}^{N}-\mathbf{G}_{z^{\prime}}^{N}\right)^{2}\right)=\rho_{N, n-5}\left(z, z^{\prime}\right) .
$$

Therefore, employing (4.4), there exists a constant $C$ such that, for $\epsilon \geq 0$,

$$
\left\{z^{\prime} \in V_{N}: d_{\infty}^{N}\left(z, z^{\prime}\right) \leq \epsilon^{2} N / C\right\} \subset B(z, \epsilon) .
$$

In particular, for $z \in V_{N}$ and $\epsilon>0$,

$$
m(B(z, \epsilon)) \geq\left(\left(\epsilon^{4} / C^{2}\right) \vee\left(1 / N^{2}\right)\right) \wedge 1 .
$$

Consequently,

$$
\int_{0}^{\infty} g(m(B(z, \epsilon))) d \epsilon \leq \int_{0}^{\sqrt{C / N}} \sqrt{\log \left(N^{2}\right)} d \epsilon+\int_{\sqrt{C / N}}^{\sqrt{C}} \sqrt{\log \left(C^{2} / \epsilon^{4}\right)} d \epsilon<C_{4},
$$

for some constant $C_{4}$. Applying Fernique's criterion (5.4), we deduce that

$$
E\left(\max _{z \in V_{N}}\left(\mathcal{S}_{z}^{N}-\overline{\mathcal{S}}_{z}^{N}\right)\right) \leq C_{4} K .
$$

The expectation $E\left(\left(\mathcal{S}_{z}^{N}-\overline{\mathcal{S}}_{z}^{N}\right)^{2}\right)$ is bounded in $N$. Therefore, using Borell's inequality, see, e.g., Ad90, Theorem 2.1], it follows that, for some constant $C_{5}$ and all $\beta>0$,

$$
P\left(\max _{z \in V_{N}}\left(\mathcal{S}_{z}^{N}-\overline{\mathcal{S}}_{z}^{N}\right) \geq C_{4} K+\beta\right) \leq 2 e^{-C_{5} \beta^{2}} .
$$


We also note the following bound, which is obtained similarly: there exist constants $C_{5}, C_{6}$ such that, for all $\beta>0$,

$$
P\left(\max _{z \in V_{N / 16}^{\prime}}\left(\overline{\mathcal{S}}_{z}^{N}-\mathcal{S}_{z}^{N / 16}\right) \geq C_{6}+\beta\right) \leq 2 e^{-C_{7} \beta^{2}} .
$$

The advantage of working with $\overline{\mathcal{S}}^{N}$ instead of $\mathcal{S}^{N}$ is that the fields $\left\{\overline{\mathcal{S}}_{z}^{N}\right\}_{z \in W_{N, i}}$ are independent for $i=1, \ldots, 4$. For every $\alpha, \beta>0$, we have the bound

$$
\begin{aligned}
& P\left(\tilde{\mathcal{S}}_{N}^{*} \geq A_{n}-\alpha\right) \\
\geq & P\left(\max _{z \in V_{N}^{\prime}} \overline{\mathcal{S}}_{z}^{N} \geq A_{n}+C_{4}-\alpha+\beta\right)-P\left(\max _{z \in V_{N}^{\prime}}\left(\mathcal{S}_{z}^{N}-\overline{\mathcal{S}}_{z}^{N}\right) \geq C_{4}+\beta\right) \\
\geq & P\left(\max _{z \in V_{N}^{\prime}} \overline{\mathcal{S}}_{N} \geq A_{n}+C_{4}-\alpha+\beta\right)-2 e^{-C_{5} \beta^{2}}
\end{aligned}
$$

where (5.5) was used in the last inequality. On the other hand, for any $\gamma, \gamma^{\prime}>0$

$$
\begin{aligned}
& P\left(\max _{z \in V_{N}^{\prime}} \overline{\mathcal{S}}_{z}^{N} \geq A_{n}-\gamma\right) \geq P\left(\max _{i=1}^{4} \max _{z \in W_{N, i}} \overline{\mathcal{S}}_{z}^{N} \geq A_{n}-\gamma\right) \\
= & 1-\left(P\left(\max _{z \in W_{N, 1}} \overline{\mathcal{S}}_{z}^{N}<A_{n}-\gamma\right)\right)^{4} \\
\geq & 1-\left(P\left(\max _{z \in V_{N / 16}^{\prime}} \mathcal{S}_{z}^{N / 16}<A_{n}-\gamma+C_{6}+\gamma^{\prime}\right)+2 e^{-C_{7}\left(\gamma^{\prime}\right)^{2}}\right)^{4},
\end{aligned}
$$

where (5.6) was used in the inequality. Combining this estimate with (5.7), we get that, for any $\alpha, \beta, \gamma^{\prime}>0$,

$$
\begin{aligned}
& P\left(\tilde{\mathcal{S}}_{N}^{*} \geq A_{n}-\alpha\right) \\
\geq & 1-2 e^{-C_{5} \beta^{2}} \\
- & \left(P\left(\max _{z \in V_{N / 16}^{\prime}} \mathcal{S}_{z}^{N / 16}<A_{n}+C_{4}+C_{6}+\beta+\gamma^{\prime}-\alpha\right)+2 e^{-C_{7}\left(\gamma^{\prime}\right)^{2}}\right)^{4} .
\end{aligned}
$$

We now iterate the last estimate. Let $\eta_{0}=1-\delta_{0}<1$ and, for $j \geq 1$, choose a constant $C_{8}=C_{8}\left(\delta_{0}\right)>0$ so that, for $\beta_{j}=\gamma_{j}^{\prime}=C_{8} \sqrt{\log \left(1 / \eta_{j}\right)}$,

$$
\eta_{j+1}=2 e^{-C_{5} \beta_{j}^{2}}+\left(\eta_{j}+2 e^{-C_{7}\left(\gamma_{j}^{\prime}\right)^{2}}\right)^{4}
$$

satisfies $\eta_{j+1}<\eta_{j}\left(1-\delta_{0}\right)$. (It is not hard to verify that such a choice is possible.) With this choice of $\beta_{j}$ and $\gamma_{j}^{\prime}$, set $\alpha_{0}=0$ and $\alpha_{j+1}=\alpha_{j}+$ $C_{4}+C_{6}+\beta_{j}+\gamma_{j}^{\prime}$, noting that $\alpha_{j} \leq C_{9} \sqrt{\log \left(1 / \eta_{j}\right)}$ for some $C_{9}=C_{9}\left(\delta_{0}\right)$. Substituting in (5.8) and using Proposition 5.2 to start the recursion, we get that

$$
P\left(\tilde{\mathcal{S}}_{N}^{*} \geq A_{n}-\alpha_{j+1}\right) \geq 1-\eta_{j+1}
$$


Therefore,

$$
\begin{aligned}
E \tilde{\mathcal{S}}_{N}^{*} & \geq A_{n}-\int_{0}^{\infty} P\left(\tilde{\mathcal{S}}_{N}^{*} \leq A_{n}-\theta\right) d \theta \\
& \geq A_{n}-\sum_{j=0}^{\infty} \alpha_{j} P\left(\tilde{\mathcal{S}}_{N}^{*} \leq A_{n}-\alpha_{j}\right) \\
& \geq A_{n}-C_{9} \sum_{j=0}^{\infty} \eta_{j} \sqrt{\log \left(1 / \eta_{j}\right)}
\end{aligned}
$$

Since $\eta_{j} \leq\left(1-\delta_{0}\right)^{j}$, it follows that there exists a constant $C_{10}>0$ so that

$$
E \mathcal{S}_{N}^{*} \geq E \tilde{\mathcal{S}}_{N}^{*} \geq A_{n}-C_{10}
$$

This completes the proof of Proposition 5.2 in the case $k_{0}=0$.

To consider the case $k_{0}>0$, define

$$
\hat{S}_{N, k_{0}}^{*}=\max _{z \in V_{N}^{\prime} \cap 2^{k_{0}} \mathbb{Z}^{2}} \mathcal{S}_{z}^{N, k_{0}} .
$$

Then, $\hat{S}_{N, k_{0}}^{*} \leq \tilde{S}_{N, k_{0}}^{*}$. On the other hand, $\hat{S}_{N, k_{0}}^{*}$ has, by construction, the same distribution as $\tilde{S}_{2^{-k_{0} N, 0}}^{*}=\tilde{S}_{2^{-k_{0} N}}^{*}$. Therefore, for any $y \in \mathbb{R}$,

$$
P\left(\tilde{S}_{N, k_{0}}^{*} \geq y\right) \geq P\left(\hat{S}_{N, k_{0}}^{*} \geq y\right) \geq P\left(\tilde{S}_{2^{-k_{0} N}}^{*} \geq y\right) .
$$

We conclude that

$$
E S_{N, k_{0}}^{*} \geq E \tilde{S}_{N, k_{0}}^{*} \geq E \tilde{S}_{2^{-k_{0} N}}^{*}
$$

Application of (5.10) completes the proof of Proposition 5.2 .

\section{Proof of Proposition 5.3}

The proof is based on the second moment method and is very similar to the argument in $[\mathrm{Br} 78$, Section 6$]$. We begin by introducing some notation. Recall that, for $z \in V_{N}$,

$$
\mathcal{S}_{z}^{N}=\sum_{k=0}^{n} \sum_{B \in \mathcal{B}_{k}(z)} b_{k, B}^{N} .
$$

We introduce a time parameter, setting

$$
\mathcal{S}_{z}^{N}(j)=\sum_{k=n-j}^{n} \sum_{B \in \mathcal{B}_{k}(z)} b_{k, B}^{N}
$$

for $j=0, \ldots, n$. Fix a (large) constant $c_{5}$ and introduce the function $L_{n}(j)$, $j=0,1, \ldots, n$, with $L_{n}(0)=L_{n}(n)=0$ and

$$
L_{n}(j)= \begin{cases}c_{5} \log j, & j=1, \ldots,\lfloor n / 2\rfloor \\ c_{5} \log (n-j), & j=\lfloor n / 2\rfloor+1, \ldots, n-1 .\end{cases}
$$


We next introduce events involving the path $\mathcal{S}_{z}^{N}(\cdot)$. Recall that $A_{n}=$ $(2 \sqrt{\log 2}) n-(3 /(4 \sqrt{\log 2})) \log n$. For $z \in V_{n}$, define the event

$\mathcal{C}_{z}=\left\{\mathcal{S}_{z}^{N}(j) \leq \frac{j}{n}\left(A_{n}+1\right)-L_{n}(j)+1, j=0,1, \ldots, n, \mathcal{S}_{z}^{N} \in\left[A_{n}, A_{n}+1\right]\right\}$.

Define

$$
h=\sum_{z \in V_{N}^{\prime}} \mathbf{1}_{\mathcal{C}_{z}}
$$

We have the following proposition.

Proposition 6.1. There exists a constant $C_{11}>0$ such that

$$
E h \geq C_{11}^{-1}
$$

and

$$
E h^{2} \leq C_{11}
$$

Proposition 5.3 follows at once from Proposition 6.1, the Cauchy-Schwartz inequality $P(h \geq 1) \geq(E h)^{2} / E\left(h^{2}\right)$, and the inequality

$$
P\left(\tilde{S}_{N}^{*} \geq A_{n}\right) \geq P(h \geq 1) .
$$

The rest of this section is devoted to the proof of Proposition 6.1 In the proof, certain crucial estimates (Lemmas 6.2 and 6.3) are discrete analogues of corresponding results in $\mathrm{Br} 78$. We provide in the appendix some detail on the proof of these lemmas.

Proof of Proposition 6.1 We begin with the following lemma; the appendix supplies details on the proof.

Lemma 6.2. For some $C_{12}, C_{13}>0$,

$$
C_{12} N^{-2} \geq P\left(\mathcal{C}_{z}\right) \geq C_{13} N^{-2} .
$$

It follows from Lemma 6.2 that

$$
E h \geq C_{12}\left|V_{N}^{\prime}\right| /\left|V_{N}\right|=C_{12} / 4,
$$

proving (6.2).

To compute the second moment in (6.3), we first set

$$
r\left(z, z^{\prime}\right)=n-\left\lceil\log _{2}\left(d_{N}^{\infty}\left(z, z^{\prime}\right)+1\right)\right\rceil,
$$

for $z, z^{\prime} \in V_{N}^{\prime}$. A crucial observation is that, by construction,

the process $\left\{\mathcal{S}_{z^{\prime}}^{N}\left(\ell+r\left(z, z^{\prime}\right)\right)-\mathcal{S}_{z^{\prime}}^{N}\left(r\left(z, z^{\prime}\right)\right)\right\}_{\ell \geq 0}$ is independent of the sigma algebra generated by the processes $\left\{\mathcal{S}_{z}^{N}(j)\right\}_{j \geq 0}$ and $\left\{\mathcal{S}_{z^{\prime}}^{N}(j)\right\}_{j \leq r\left(z, z^{\prime}\right)}$. 
(Note that the boxes involved in the construction of the first process are disjoint from those of the other two processes.) We employ the decomposition

$$
\begin{aligned}
E h^{2} & =\sum_{z, z^{\prime} \in V_{N}^{\prime}} P\left(\mathcal{C}_{z}, \mathcal{C}_{z^{\prime}}\right) \\
& =\sum_{z, z^{\prime}: r\left(z, z^{\prime}\right)<n / 2} P\left(\mathcal{C}_{z}, \mathcal{C}_{z^{\prime}}\right)+\sum_{z, z^{\prime}: r\left(z, z^{\prime}\right) \geq n / 2} P\left(\mathcal{C}_{z}, \mathcal{C}_{z^{\prime}}\right)=: Q_{1}+Q_{2} .
\end{aligned}
$$

We begin by considering $Q_{2}$. For this, we introduce the event $\tilde{\mathcal{C}}_{z, z^{\prime}}=\left\{\mathcal{S}_{z^{\prime}}^{N}\left(r\left(z, z^{\prime}\right)\right) \leq \frac{r\left(z, z^{\prime}\right)}{n}\left(A_{n}+1\right)-L_{n}\left(r\left(z, z^{\prime}\right)\right)+1, \mathcal{S}_{z^{\prime}}^{N} \in\left[A_{n}, A_{n}+1\right]\right\}$, noting that $\mathcal{C}_{z^{\prime}} \subset \mathcal{C}_{z, z^{\prime}}$. It follows from (6.5) that $P\left(\mathcal{C}_{z}, \mathcal{C}_{z^{\prime}}\right) \leq P\left(\mathcal{C}_{z}, \tilde{\mathcal{C}}_{z, z^{\prime}}\right)$

$$
\leq P\left(\mathcal{C}_{z}\right) P\left(G_{z, z^{\prime}} \geq\left(1-\frac{r\left(z, z^{\prime}\right)}{n}\right)\left(A_{n}+1\right)+L_{n}\left(r\left(z, z^{\prime}\right)\right)\right),
$$

where $G_{z, z^{\prime}}$ is a centered Gaussian random variable of variance

$$
n-r\left(z, z^{\prime}\right)=\left\lceil\log _{2}\left(d_{N}^{\infty}\left(z, z^{\prime}\right)+1\right)\right\rceil=: u\left(z, z^{\prime}\right) .
$$

Therefore, using (6.4),

$$
P\left(\mathcal{C}_{z}, \mathcal{C}_{z^{\prime}}\right) \leq C_{14} N^{-2} \exp \left(-\left(\left(A_{n} / n\right) u\left(z, z^{\prime}\right)+L_{n}\left(r\left(z, z^{\prime}\right)\right)\right)^{2} / 2 u\left(z, z^{\prime}\right)\right)
$$$$
\text { Since the number of points } z^{\prime} \text { with } d_{N}^{\infty}\left(z, z^{\prime}\right) \in\left[2^{k}, 2^{k+1}\right] \text { is bounded by a }
$$
constant multiple of $2^{2 k}$, we conclude from (6.7) that

$$
Q_{2} \leq C_{16} \sum_{k=n / 2}^{n}(n-k)^{-c_{5} A_{n} / n} n^{3}<C_{17},
$$

if $c_{5}$ is chosen large enough.

It thus remains to handle $Q_{1}$. Introduce the events

$$
\mathcal{D}_{z, z^{\prime}}^{(1)}=\left\{\mathcal{S}_{z^{\prime}}^{N}\left(r\left(z, z^{\prime}\right)\right) \leq \frac{r\left(z, z^{\prime}\right)}{n}\left(A_{n}+1\right)-L_{n}\left(r\left(z, z^{\prime}\right)\right)+1\right\}
$$

and, for $w \in \mathbb{R}$,

$$
\begin{aligned}
\mathcal{D}_{z, z^{\prime}, w}^{(2)}= & \left\{\mathcal{S}_{z^{\prime}}^{N}(j)-\mathcal{S}_{z^{\prime}}^{N}\left(r\left(z, z^{\prime}\right)\right) \leq \frac{j}{n}\left(A_{n}+1\right)-w+1, j=r\left(z, z^{\prime}\right), \ldots, n,\right. \\
& \left.\mathcal{S}_{z^{\prime}}^{N}(n)-\mathcal{S}_{z^{\prime}}^{N}\left(r\left(z, z^{\prime}\right)\right) \in\left[A_{n}-w, A_{n}+1-w\right]\right\} .
\end{aligned}
$$

It follows again from (6.5) that

$$
\begin{aligned}
P\left(\mathcal{C}_{z}, \mathcal{C}_{z^{\prime}}\right) & \leq P\left(\mathcal{C}_{z}, \mathcal{D}_{z, z^{\prime}}^{(1)}, \mathcal{D}_{z, z^{\prime}, \mathcal{S}_{z^{\prime}}^{N}\left(r\left(z, z^{\prime}\right)\right)}^{(2)}\right) \\
& \leq P\left(\mathcal{C}_{z}\right)_{w \leq r\left(z, z^{\prime}\right)\left(A_{n}+1\right) / n-L_{n}\left(r\left(z, z^{\prime}\right)\right)+1} P\left(\mathcal{D}_{z, z^{\prime}, w}^{(2)}\right)
\end{aligned}
$$

To analyze $P\left(\mathcal{D}_{z, z^{\prime}, w}^{(2)}\right)$, we employ the following lemma. (Details of the proof are given in the appendix.) 
Lemma 6.3. With notation as above, there exist constants $C_{19}$ and $C_{20}$ so that, if $r\left(x, x^{\prime}\right) \leq n / 2$ and $w \leq r\left(z, z^{\prime}\right)\left(A_{n}+1\right) / n-L_{n}\left(r\left(z, z^{\prime}\right)\right)+1$, then

$$
P\left(\mathcal{D}_{z, z^{\prime}, w}^{(2)}\right) \leq C_{20}\left(L\left(r\left(z, z^{\prime}\right)\right)+1\right) \cdot 2^{-2 \log _{2} d_{N}^{\infty}\left(z, z^{\prime}\right)} \cdot e^{-C_{19} L\left(r\left(z, z^{\prime}\right)\right)} .
$$

Substituting (6.9) of the lemma into (6.8), we conclude that

$$
Q_{1} \leq C_{20} \sum_{k=0}^{n / 2}(L(k)+1) e^{-C_{19} L(k)} \leq C_{21} .
$$

This completes the proof of Proposition 6.1.

\section{Appendix}

In this appendix, we provide more detail on the bounds in (6.4) of Lemma 6.2 and (6.9) of Lemma 6.3, which in both cases are very similar to material in $\mathrm{Br} 78$.

Bounds in Lemma 6.2. Using the Brownian bridge $\mathfrak{z}(s), s \in[0, n]$, that is standard Brownian motion $\mathfrak{x}(s), s \geq 0$, conditioned on $\mathfrak{x}(n)=0$, one has (A.1) $P(\mathfrak{z}(s)<2, s=0, \ldots, n) \geq P\left(\mathcal{C}_{z}\right) / K(n) \geq P(\mathfrak{z}(s)<1-L(s), s=0, \ldots, n)$, where $K(n)=P\left(\mathfrak{x}(n) \in\left[A_{n}, A_{n}+1\right]\right.$. One can check that $\left(N^{2} / n\right) K(n) \in$ $\left[C_{22}, C_{23}\right]$, for $0<C_{22}<C_{23}<\infty$. So, in order to demonstrate Lemma 6.2, it suffices to show the bounds on each side of (A.1) are of order $1 / n$.

By [Br78, Proposition $2^{\prime}$ on page 555],

$$
P(\mathfrak{z}(s)<1-L(s), s \in[0, n]) \geq C_{24} / n,
$$

which gives the desired lower bound. One obtains the analogous upper bound $C_{25} / n$ for the left side of (A.1) by applying the reflection principle to Brownian bridge; see also [Br78, Lemma 9]. (The bound in discrete time is the same as that in continuous time, up to the constant $C_{25}$, since the "overshoot" of the normal past a boundary, over a unit time interval, has bounded second moment.)

Bound in Lemma 6.3. The bound in (6.9) is obtained in the same manner as are parts (a) and (b) of Lemma 11 on page 565 of $[\mathrm{Br} 78$. One can apply the reflection principle as in part (a), but for discrete time instead of continuous time. (As in the derivation of the upper bound of (6.4), the "overshoot" of the normal only affects the constant in front.) One obtains with a little work that, for $w \leq r\left(z, z^{\prime}\right)\left(A_{n}+1\right) / n-L_{n}\left(z, z^{\prime}\right)+1$,

$$
P\left(D_{z, z^{\prime}, w}^{(2)}\right) \leq C_{26} n^{-\frac{3}{2}}\left(-w+\frac{r\left(z, z^{\prime}\right)}{n} A_{n}+2\right) \exp \left\{-\left(A_{n}-w\right)^{2} / 2 u\left(z, z^{\prime}\right)\right\} .
$$

As in part (b) of Lemma 11 of [Br78], for the above range of $w$, the right side of (A.3) is maximized at the boundary $w=r\left(z, z^{\prime}\right)\left(A_{n}+1\right) / n-$ $L_{n}\left(z, z^{\prime}\right)+1$. Plugging this value of $w$ into the right side of (A.3) yields (6.9). 


\section{REFERENCES}

[ABR09] L. Addario-Berry and B. Reed, Minima in branching random walks, Annals Probab. 37 (2009), pp. 1044-1079.

[Ad90] R. Adler, An Introduction to Continuity, Extrema and Related Topics for General Gaussian Processes, Lecture Notes - Monograph Series, Institute Mathematical Statistics, Hayward, CA (1990).

[BDG01] E. Bolthausen, J.-D. Deuschel and G. Giacomin, Entropic repulsion and the maximum of the two-dimensional harmonic crystal, Ann. Probab. 29 (2001), pp. 1670-1692.

[BDZ10] E. Bolthausen, J.-D. Deuschel and O. Zeitouni, Recursions and tightness for the maximum of the discrete, two dimensional Gaussian Free Field. Submitted. http://arxiv.org/pdf/1005.5417v1

[Br78] M. Bramson, Maximal displacement of branching Brownian motion, Comm. Pure Appl. Math. 31 (1978), pp. 531-581.

[Br83] M. Bramson, Convergence of Solutions of the Kolmogorov Equation to Travelling Waves, Mem. Amer. Math. Soc. 44 (1983), \#285.

[CLD01] D. Carpentier and P. Le Doussal, Glass transition for a particle in a random potential, front selection in nonlinear renormalization group, and entropic phenomena in Liouville and Sinh-Gordon models, Phys. Rev. E 63 (2001), 026110.

[Ch08] S. Chatterjee, Chaos, concentration, and multiple valleys, arXiv:0810.4221 v2.

[DH91] F. M. Dekking and B. Host, Limit distributions for minimal displacement of branching random walks, Probab. Theory Relat. Fields 90 (1991), pp. 403-426.

[Fe75] X. Fernique, Regularité des trajectoires des fonctions aléatoires gaussiennes, Ecole d'Eté de Probabilités de Saint-Flour, IV-1974, pp. 1-96. Lecture Notes in Math. 480, Springer, Berlin, (1975).

[La91] G. Lawler, Intersections of Random Walks, Birkhäuser, Boston (1991).

Maury Bramson

School of Mathematics

University of Minnesota

206 Church St. SE

Minneapolis, MN 55455

bramson@math.umn.edu
Ofer Zeitouni

School of Mathematics

University of Minnesota

206 Church St. SE

Minneapolis, MN 55455

and

Faculty of Mathematics

Weizmann Institute of Science

Rehovot 76100, Israel

zeitouni@math.umn.edu 